\title{
Uncertainty in ultrasound reporting
}

\author{
Anil Kapoor, MD, FRCSC
}

See related article on page 251

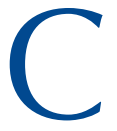

hung and colleagues present an interesting study on the high discordance rate between community and hospital ultrasound reports for urologic abnormalities. ${ }^{1}$ This retrospective chart review found a discordance rate of $52.2 \%$ in community ultrasound reports followed by hospital ultrasound findings. A discordance rate of $43.3 \%$ was found with community ultrasound reports followed by hospital CT scans. In total, the discrepancy rate between hospital and community studies was $48.4 \%$. Conversely, the discordance rate between hospital ultrasound and hospital CT scan reports was $0 \%$; that is, hospital CT scan findings agreed with hospital ultrasound findings in all cases.

The authors acknowledge the limitations of the study, including temporal bias, as the hospital studies were all performed after the community studies. There is variation in quality and expertise of the community ultrasound sites and technologists, which was not stratified.

In many provinces, such as Alberta and Quebec, it is mandated that a radiologist be on site at all times during the provision of ultrasound. This is not a requirement in Ontario (where the study was performed) in that a radiologist has to be available but not on site at the time of the ultrasound examination. However, in this study it is assumed that the radiologist was not present during any of the community ultrasounds, when in fact a radiologist may have been called in for at least some of these community ultrasounds. The variability in ultrasound technician experience in these community centres may also have a profound impact.

This does not diminish the surprising discordance between community ultrasound examinations and hospital examinations in this study.
This up to $48 \%$ discordance creates not a trivial increase in cost to the system associated with repetition of radiologic studies, anxiety and confusion for patients and doctors over uncertain results. To correct this discordance, it remains to be proven whether the confounding factors are lack of quality equipment, ultrasound technician experience or the presence of an on-site radiologist. Certainly this discordance between community and hospital radiology centres is unacceptable and requires further study.

Associate Professor of Surgery (Urology), McMaster University, Hamilton, Ont.

This article has been peer reviewed.

Competing interests: None declared.

\section{Reference}

1. Chung DE, Platzker T, Chung J, et al. Discordance of community and hospital ultrasound reports for urological abnormalities. CUAJ 2007; 1:251-4.

Correspondence: Dr. Anil Kapoor, McMaster Institute of Urology, St. Joseph's Healthcare, 50 Charlton Ave. E., Hamilton ON L8N 4A6; kapoor4 @mcmaster.ca 